\title{
A NOTE ON CONNECTED AND PERIPHERALLY CONTINUOUS FUNCTIONS
}

\begin{abstract}
MELVIN R. HAGAN
ABSTRACT. In this paper it is proved that under certain conditions on the domain and range spaces an open monotone connected function preserves unicoherentness and hereditary local connectedness. In addition, a monotone-light factorization theorem is proved for certain connected functions and peripherally continuous functions.
\end{abstract}

1. Introduction. Many properties of continuous functions are also possessed by certain noncontinuous functions (see for example [1][4] and [6]). This paper is concerned with three of these properties: namely, preservation by an open monotone connected function of unicoherentness and hereditary local connectedness, and factorization of connected and peripherally continuous functions.

Some definitions will now be recalled. Let $X$ and $Y$ be topological spaces. A function $f: X \rightarrow Y$ is connected if whenever $A$ is a connected set in $X$, then $f(A)$ is connected in $Y[6$, p. 488]. The function $f$ is peripherally continuous if whenever $p$ is a point in $X$ and $U$ and $V$ are open sets containing $p$ and $f(p)$, respectively, there exists an open set $W$ such that $x \in W \subset U$ and $f$ maps the boundary of $W$ into $V[2, \mathrm{p}$. 751]. The function $f$ is monotone if for each $y \in Y, f^{-1}(y)$ is connected.

If $A$ is a set, $\operatorname{cl}(A)$ will denote the closure of $A$ and $F(A)$ will denote the boundary of $A$. The notation $x_{n} \rightarrow x$ is used for a sequence $\left\{x_{n}\right\}$ converging to $x$. If $A$ is a collection of sets, $A^{*}$ will denote the point set union of the sets in $A$.

2. Connected functions. In Theorems 1 and 2 the only assumptions concerning the function $f$ are that $f$ is open in Theorem 1 and $f$ is open and monotone in Theorem 2. These theorems are then used in Theorems 3 and 4 to obtain the results that, under certain conditions, unicoherentness and hereditary local connectedness are preserved by an open monotone connected function.

Presented to the Society, March 25, 1970 under the title Some properties of open, monotone, connected functions; received by the editors January 20, 1970.

AMS 1969 subject classifications. Primary 5460; Secondary 5455.

Key words and phrases. Open monotone connected function, peripherally continuous function, unicoherent continuum, hereditarily locally connected continuum, upper semicontinuous decomposition, monotone-light factorization. 
THEOREM 1. If $f$ is an open function from the topological space $X$ onto the topological space $Y$ and $y_{n} \rightarrow y$ in $Y$, then $f^{-1}(y) \subset \lim \inf f^{-1}\left(y_{n}\right)$.

Proof. If $x \in f^{-1}(y)$ and $U$ is an open set in $X$ such that $x \in U$ and $U$ fails to intersect $f^{-1}\left(y_{n}\right)$ for infinitely many $n$, then since $f$ is open $f(U)$ is an open set containing $y$ and $f(U)$ fails to contain $y_{n}$ for infinitely many $n$. This contradicts $y_{n} \rightarrow y$.

THEOREM 2. If $f$ is an open monotone function from the topological space $X$ onto the first countable space $Y$ and $E$ is connected in $Y$, then $f^{-1}(E)$ is connected in $X$.

Proof. Suppose $f^{-1}(E)=H \cup K$, where $H$ and $K$ are separated. Then $E=f(H) \cup f(K)$ and $f(H)$ and $f(K)$ are not separated. Suppose $q$ is a point in $f(H) \cap f(K)$. Then for some $r$ in $H$ and $t$ in $K, f(r)=f(t)=q$. Hence $r$ and $t$ are in $f^{-1}(q)$. Since $f$ is monotone $f^{-1}(q)$ is connected. Therefore either $f^{-1}(q) \subset H$ or $f^{-1}(q) \subset K$ since $H$ and $K$ are separated. But $r \in f^{-1}(q) \subset H$ and $t \in f^{-1}(q) \subset K$. This contradiction shows that $f(H) \cap f(K)=\varnothing$. Thus, one of $f(H)$ and $f(K)$ must contain a limit point of the other. Suppose $q$ is in $f(H)$ and is a limit point of $f(K)$. Since $Y$ is first countable there is a sequence $\left\{q_{n}\right\}$ in $f(K)$ such that $q_{n} \rightarrow q$. By Theorem $1, f^{-1}(q) \subset \lim$ inf $f^{-1}\left(q_{n}\right)$. Hence, if $x$ is in $f^{-1}(q)$, then $x$ is a limit point of $K$ since $f^{-1}\left(q_{n}\right) \subset K$ for all $n$. But $x \in H$ since $f^{-1}(q) \subset H$. Thus, $H$ and $K$ are not separated. This contradiction shows that $f^{-1}(E)$ is connected.

THEOREM 3. If $f$ is an open monotone connected function from the unicoherent $T_{2}$ continuum $X$ onto the first countable compact $T_{2}$ space $Y$, then $Y$ is a unicoherent continuum.

Proof. Since $f$ is a connected function, then $Y$ is connected and hence a continuum by the virtue of being compact. Suppose $Y=H \cup K$, where $H$ and $K$ are continua. Then $X=f^{-1}(H) \cup f^{-1}(K)$. By Theorem $2, f^{-1}(H)$ and $f^{-1}(K)$ are connected. By Theorem 2.1 of [4], $f^{-1}(H)$ and $f^{-1}(K)$ are closed and hence are continua since $X$ is compact. Since $X$ is unicoherent $f^{-1}(H) \cap f^{-1}(K)$ is a continuum. Now $f^{-1}(H) \cap f^{-1}(K)=f^{-1}(H \cap K)$ and since $f$ is connected, $f\left(f^{-1}(H \cap K)\right)=H \cap K$ is connected. Thus, $H \cap K$ is a closed connected subset of the compact space $Y$ and is therefore a continuum. This shows that $Y$ is unicoherent.

THEOREM 4. If $f$ is an open monotone connected function from $X$ onto $Y$, where $X$ and $Y$ are separable metric continua and $X$ is hereditarily locally connected, then $Y$ is hereditarily locally connected. 
Proof. By Theorem 2.1 of $[7$, p. 89] a continuum is hereditarily locally connected if and only if it has no nondegenerate continuum of convergence. Therefore, suppose $K$ is a nondegenerate continuum of convergence in $Y$. Let $\left\{K_{n}\right\}$ be a sequence of disjoint continua none intersecting $K$ such that $\lim K_{n}=K$, and let $p$ and $q$ be distinct points in $K$. By Theorem 7 of [7, p.11] some subsequence $\left\{f^{-1}\left(K_{n i}\right)\right\}$ of $\left\{f^{-1}\left(K_{n}\right)\right\}$ converges to a limiting set $L$. Since $X$ is compact, $L \neq \varnothing$. Hence, by Theorem 9.1 of [7, p. 14], $L$ is a continuum. Let $x \in f^{-1}(p)$ and $U$ an open set in $X$ containing $x$. Since $f$ is an open function, $f(U)$ is open and contains $p$. Therefore $f(U)$ intersects $K_{n}$ for all but a finite number of $n$. Hence, $U$ intersects $f^{-1}\left(K_{n}\right)$ for all but a finite number of $n$, and consequently intersects $f^{-1}\left(K_{n i}\right)$ for all but a finite number of $i$. Thus, $x \in L$ and hence $f^{-1}(p) \subset L$. Similarly, $f^{-1}(q) \subset L$. Since neither of $f^{-1}(p)$ and $f^{-1}(q)$ is empty and they are disjoint, it follows that $L$ is nondegenerate. Therefore $X$ contains a nondegenerate continuum of convergence. This contradicts $X$ being hereditarily locally connected. Thus, $Y$ is hereditarily locally connected.

3. Factorization. If $f$ is a function from the space $X$ onto the space $Y$, let $X^{\prime}$ denote the collection of all components of sets $f^{-1}(y)$, where $y$ varies over $Y$. The collection $X^{\prime}$ will be called the component decomposition of $X$ induced by $f$. Theorems 5 and 6 give a factorization of $f$ analogous to that given in Theorems 3.6 and 3.7 of [1].

THEOREM 5. If $f$ is a connected function from the compact, separable metric space $X$ onto the regular $T_{2}$ space $Y$, and the component decomposition $X^{\prime}$ of $X$ induced by $f$ is upper semicontinuous, then $f$ can be factored into the composite $f=f_{2} f_{1}$, where $f_{1}$ from $X$ onto $X^{\prime}$ is monotone and continuous and $f_{2}$ from $X^{\prime}$ onto $Y$ is light and connected.

Proof. Define $f_{1}(x)=C$ if and only if $x$ is a point in $C$, where $C$ is a component of some $f^{-1}(y), y$ in $Y$. By [7, p. 127], $f_{1}$ is monotone and continuous. Define $f_{2}(C)=y$ if and only if $C$ is a component of $f^{-1}(y)$. Then $f_{2}$ is light since the elements of $f_{2}^{-1}(y)$ are the components of $f^{-1}(y)$ and these form a totally disconnected set in $X^{\prime}$. For if $H$ is any nondegenerate subcollection of $f_{2}^{-1}(y)$, then $H$ is connected in $X^{\prime}$ if and only if $H^{*}$ is connected in $X$ [5, p. 275]. Now $H$ being nondegenerate implies $H^{*}$ contains more than one component of $f^{-1}(y)$ and hence is not connected. Thus $H$ is not connected and $f_{2}$ is therefore light.

By definition of $f_{1}$ and $f_{2}, f=f_{2} f_{1}$. Therefore it remains to show that $f_{2}$ is connected.

To this end let $A$ be a connected subset of $X^{\prime}$. Then $A^{*}$ is connected in $X$. Since $f$ is a connected function $f\left(A^{*}\right)$ is connected. But $f\left(A^{*}\right)$ 
$=f_{2} f_{1}\left(A^{*}\right)=f_{2}(A)$. Hence, $f_{2}(A)$ is connected and $f_{2}$ is a connected function.

THEOREM 6. If $f$ is a peripherally continuous function from the compact separable metric space $X$ onto the regular $T_{2}$ space $Y$ and the component decomposition $X^{\prime}$ of $X$ is upper semicontinuous, then $f$ can be factored into the composite $f=f_{2} f_{1}$, where $f_{1}$ and $f_{2}$ are defined as in Theorem $5, f_{1}$ is monotone and continuous, and $f_{2}$ is light and peripherally continuous.

Proof. That $f_{1}$ is monotone and continuous and $f_{2}$ is light follows as in Theorem 5 .

Since $f$ is peripherally continuous, the components of $f^{-1}(y), y$ in $Y$, are closed by Theorem 1 of $\left[3\right.$, p. 639]. Hence the elements of $X^{\prime}$ are continua since $X$ is compact.

Let $g$ be an element in $X^{\prime}$ and $f(g)=y$, and let $U$ and $V$ be open in $X^{\prime}$ and $Y$ containing $g$ and $y$, respectively. Then $U^{*}$ is open in $X$ and $g \subset U^{*}$. Since $f$ is peripherally continuous, for each $x$ in $g$ there is an open set $W_{x}$ in $X$ such that $x \in W_{x} \subset U^{*}$ and $f\left(F\left(W_{x}\right)\right) \subset V$. The collection $\left\{W_{x}: x \in g\right\}$ is an open covering of $g$. Since $g$ is compact some finite subcollection $\left\{W_{1}, \cdots, W_{n}\right\}$ covers $g$. Let $W=\bigcup_{i=1}^{n} W_{i}$. Then $g \subset W \subset U^{*}$ and $f(F(W)) \subset V$.

Let $H \subset X^{\prime}$ such that $k$ is in $H$ if and only if $k \subset W$. Then $H$ is open in $X^{\prime}$ and $H \subset U$. Let $h \in F(H)$ and suppose $h \cap F(W)=\varnothing$. Now $h \subset \operatorname{cl}(W)=W \cup F(W)$ and $W \cap F(W)=\varnothing$. Hence, $h \subset W$ and thus $h \in H$. This contradicts $h \in F(H)$. Therefore, $h \cap F(W) \neq \varnothing$. Let $x \in h \cap F(W)$. Then $f(x)=f(h)=f_{2}(h)$ and since $x \in F(W), f(x) \in V$. Thus, $f_{2}(h) \in V$ and $f_{2}(F(H)) \subset V$. This shows that $f_{2}$ is peripherally continuous.

REMARK. In [1] a proof is given that under rather restrictive conditions on the space $X$, the component decomposition $X^{\prime}$ of $X$ induced by a peripherally continuous function is indeed upper semicontinuous. If this could be proven under milder restrictions for connected and peripherally continuous functions, then Theorems 5 and 6 would more closely resemble the monotone-light factorization theorem for continuous functions [7, p. 143].

The author wishes to acknowledge helpful comments made by the referee.

\section{REFERENCES}

1. M. R. Hagan, Upper semi-continuous decompositions and factorization of certain non-continuous transformations, Duke Math. J. 32 (1965), 679-687. MR 32 \#4668. 
2. O. H. Hamilton, Fixed points for certain noncontinuous transformations, Proc. Amer. Math. Soc. 8 (1957), 750-756. MR 19, 301.

3. P. E. Long, Properties of certain non-continuous transformations, Duke Math. J. 28 (1961), 639-645. MR 24 \#A2945.

4. - Connected mappings, Duke Math. J. 35 (1968), 677-682. MR 38 \#2745.

5. R. L. Moore, Foundations of point set theory, rev. ed., Amer. Math. Soc. Colloq. Publ., vol. 13, Amer. Math. Soc., Providence, R.I., 1962. MR 27 \#709.

6. W. J. Pervin and Norman Levine, Connected mappings of Hausdorff spaces, Proc. Amer. Math. Soc. 9 (1958), 488-496. MR 20 \#1970.

7. G. T. Whyburn, Analytic topology, Amer. Math. Soc. Colloq. Publ., vol. 28, Amer. Math. Soc., Providence, R.I., 1963. MR 32 \#425.

North Texas State University, Denton, Texas 76201 